\title{
Three-dimensional ZnO/Si broom-like nanowire heterostructures as photoelectrochemical anodes for solar energy conversion
}

\author{
Alireza Kargar', Ke Sun', Sung Joo Kim², Dylan Lu', Yi Jing', Zhaowei Liu', Xiaoqing Pan², and Deli Wang ${ }^{*, 1,3,4}$ \\ ${ }^{1}$ Department of Electrical and Computer Engineering, University of California, San Diego, 9500 Gilman Drive, La Jolla, \\ California 92093, USA \\ ${ }^{2}$ Department of Materials Science and Engineering, University of Michigan, 2300 Hayward Street, Ann Arbor, Michigan 48109, USA \\ ${ }^{3}$ Materials Science and Engineering Program, University of California, San Diego, 9500 Gilman Drive, La Jolla, California 92093, USA \\ ${ }^{4}$ California Institute of Telecommunication and Information Technology, University of California, San Diego, 9500 Gilman Drive, La Jolla, \\ California 92093, USA
}

Received 31 March 2013, revised 20 June 2013, accepted 23 July 2013

Published online 4 September 2013

Keywords heterostructures, nanowires, photoelectrochemical cells, silicon, solar energy conversion, water oxidation, water splitting, $\mathrm{ZnO}$

*Corresponding author: e-mail deliwang@eng.ucsd.edu, Phone: +1 858822 4723, Fax: +1 8585340556

We report a low-cost solution fabrication of three-dimensional (3D) $\mathrm{ZnO} / \mathrm{Si}$ broom-like nanowire (NW, "nanobroom") heterostructures, consisting of $\mathrm{Si} \mathrm{NW}$ "backbones" and $\mathrm{ZnO}$ NW "stalls", and their application as photoelectrochemical anodes for solar water splitting and energy conversion. The nanobroom morphology and atomic structure are characterized using the scanning, transmission, and scanning transmission electron microscopies. Both Si NW backbones and ZnO NW stalls are defect-free, single-crystalline, and their surfaces are smooth. The optical absorption and photocurrents from nanobroom array electrodes with different $\mathrm{Si}$ and $\mathrm{ZnO} \mathrm{NW}$ dimensions are studied. The longer Si NW backbones and smaller $\mathrm{ZnO} \mathrm{NW}$ stalls lead to better light absorption and larger photoanodic current. The $\mathrm{ZnO} / \mathrm{Si}$ nanobrooms show much higher photoanodic current than the bare Si NWs due to the effective $\mathrm{Si} / \mathrm{ZnO}$ junction and increased surface area. The nanobroom electrode stability is also investigated and using a thin $\mathrm{TiO}_{2}$ coating layer protecting the NWs against dissolution, long-term stability is obtained without any change in shape and morphology of nanobrooms. Finally, the effect of catalyst to improve the oxygen evolution reaction (OER) at the electrode surface is studied resulting in large enhancement in photoanodic current and significant reduction in anodic turn-on potential. This study reveals the promise of the use of simply fabricated and low-cost 3D heterostructured NW photoelectrodes for clean solar energy harvesting and conversion.
1 Introduction Direct solar water splitting to generate hydrogen using photoelectrochemical (PEC) cells, as a costeffective and carbon-emission-free hydrogen production method, has attracted considerable attention [1, 2]. Solar water splitting has great potential for practical clean hydrogen production [3, 4]. Efficient photocathodes and photoanodes are required to couple to each other to perform overall solar water splitting without any external bias [2]. Semiconductor photoelectrodes are the key components to determine the PEC performance and solar energy harvesting/ conversion efficiency. Among the semiconductor materials studied, nanowire (NW) heterostructures have been of great interest due to the potential of superior efficiency coming from the unique geometry and properties, unlimited combination and effective integration at nanoscale to implement design, and material integrity [5, 6]. Particularly core/shell [5-7] and branched NW heterostructures [8] have been reported to offer higher efficiency compared to pure semiconductor NWs or bulk materials because of the enhanced absorption in broad solar spectra, improved charge separation and reduced recombination of photo-generated carriers, and increased charge transportation. Moreover, branched NW heterostructures can provide better PEC performance and higher solar-to-fuel production efficiency than the core/shell NW heterojunctions due to much increased surface reaction area and improved gas evolution 
coming from the large surface curvature of NW branches [8, 9]. We have recently demonstrated efficient, stable, and costeffective three-dimensional (3D) $\mathrm{n}-\mathrm{ZnO} / \mathrm{p}-\mathrm{Si}$ branched $\mathrm{NW}$ photocathodes with low photocathodic turn-on potential for water reduction and hydrogen gas production $[9,10]$. This paper reports the low-cost fabrication and systematic characterization of 3D n- $\mathrm{ZnO} / \mathrm{n}$-Si broom-like NW (nanobroom) heterostructures, with $\mathrm{n}-\mathrm{ZnO} \mathrm{NWs}$ only covering the tip of n-Si NWs, and their application as photoanodes for water oxidation and oxygen gas generation. The optical absorption and photocurrent, as well as the electrode stability, of the nanobroom photoelectrodes are studied and the PEC performances are optimized based on different $\mathrm{Si}$ and $\mathrm{ZnO}$ NWs dimensions. The effect of the catalyst on the PEC performance of nanobrooms is also investigated.

2 Experimental A schematic for the fabrication procedure of $\mathrm{n}-\mathrm{ZnO} / \mathrm{n}-\mathrm{Si}$ nanobrooms is shown in Fig. S1 of Supporting Information. Firstly, wafer-scale vertically ordered Si NW arrays on Si wafer were obtained using an aqueous Ag-assisted electroless etching method [11, 12]. Ntype phosphorus-doped silicon (100) wafers with resistivity of $1-20 \Omega \mathrm{cm}$ were first cleaned with solvent, rinsed with deionized (DI) water, and dried with $\mathrm{N}_{2}$ flow. The cleaned $\mathrm{Si}$ wafer was then fixed on a homemade etching setup, and after that the setup with the attached wafer was submerged in a mixture solution of $\mathrm{HF}$ and $\mathrm{AgNO}_{3}$ solution. The etching solution container was then put in a water bath at a temperature $50^{\circ} \mathrm{C}$. The wafers were then etched for different times to achieve different lengths of Si NWs. The etched wafer was then taken out of etching solution and rinsed with DI water. Finally, it was submerged in a 1:10 diluted $\mathrm{HNO}_{3}$ solution for $2 \mathrm{~h}$ to remove the $\mathrm{Ag}$ dendrite film formed during the etching. The samples were denoted by the etching time (e.g., "10 min Si NWs" means the Si NW backbones that were etched for $10 \mathrm{~min}$ ).

Secondly, the etched Si NW samples were then dipped in buffered oxide etching (BOE) solution for $10 \mathrm{~s}$ to remove the native oxide layer, cleaned with DI water, dried with $\mathrm{N}_{2}$ gun, and instantly transferred to a sputter machine in order to minimize oxidation. A thin $\mathrm{ZnO}$ seeding layer was deposited on the Si NW substrates using RF magnetron sputtering with $\mathrm{ZnO}$ target (purity; 99.99\%) in argon carrier gas. The sputtering pressure was 1.9 mTorr during the deposition.

Finally, the ZnO NW stalls were grown on the Si NWs using hydrothermal growth method reported before [10, 1214]. Briefly, $25 \mathrm{mM}$ aqueous solutions of zinc acetate dihydrate $\left(\mathrm{Zn}\left(\mathrm{O}_{2} \mathrm{CCH}_{3}\right)_{2}\left(\mathrm{H}_{2} \mathrm{O}\right)_{2}\right.$, Sigma-Aldrich $)$ and hexamethylene-tetramine (HMTA) $\left(\left(\mathrm{CH}_{2}\right)_{6} \mathrm{~N}_{4}\right.$, Sigma-Aldrich) in DI water $(\geq 17.5 \mathrm{M} \Omega \mathrm{cm})$ were prepared (growth solution). The $\mathrm{ZnO}$-seeded Si NW substrates, fixed on a supporting glass slide using double-sided tape with their top facing down, were then immersed in the growth solution which was pour in a flask. Then, the flask was positioned in the center of an oil bath and a mild agitation was used at the bottom of the flask. After reaching a temperature of $60^{\circ} \mathrm{C}$ for the growth solution (the temperature was monitored with a thermometer kept inside the growth solution), the time counting was started for different desired growth times $(5,10,15 \mathrm{~min}$ here). The as-synthesized samples were then rinsed carefully with DI water and dried with $\mathrm{N}_{2}$ gun. The samples were denoted by the growth time (e.g., " $10 \mathrm{~min} \mathrm{ZnO} \mathrm{NWs"} \mathrm{means}$ the nanobrooms with $\mathrm{ZnO} \mathrm{NW}$ stalls grown for $10 \mathrm{~min}$ ).

For the $\mathrm{TiO}_{2}$-coated samples, atomic layer deposition (ALD), using Beneq TFS 200 system, was used to deposit a thin layer of $\mathrm{TiO}_{2}$ on the nanobroom substrates at a temperature of $250{ }^{\circ} \mathrm{C}$. The measured thickness of deposited $\mathrm{TiO}_{2}$ was $10 \mathrm{~nm}$ based on growth rate of $0.44 \AA$ /cycle on $\mathrm{Si}$ (100) film substrate. For Ru catalyst/co-catalyst deposition, sputtering was used to deposit $\sim 5 \mathrm{~nm} \mathrm{Ru}$ on the samples at a sputtering pressure of $10 \mathrm{mTorr}$.

The nanobroom morphology was examined using fieldemission environmental scanning electron microscopy (SEM, Phillips XL30) and ultra-high-resolution scanning electron microscopy (UHR SEM, FEI XL30-SFEG), both at an accelerating voltage of $10.0 \mathrm{kV}$. The atomic-scale structural analyses were performed using high-resolution transmission electron microscopy (HRTEM, JEOL JEM3100F) operated at $300 \mathrm{kV}$. Spherical aberrationcorrected scanning transmission electron microscopy (Cscorr. STEM, JEOL JEM2100F) operated at $200 \mathrm{kV}$ was employed to identify the actual atomic structure of NWs.

The reflectance spectra were taken by an optical spectrometer. White light (xenon lamp $75 \mathrm{~W}$ ) was focused through a microscope objective of $50 \times$ with a numerical aperture (NA) of 0.8 onto the samples. The absorption spectra $(A)$ were achieved by reflectance spectra $(R)$ using $A$ $(\%)=100-R(\%)$.

For the PEC tests, $\mathrm{Cu}$ wire was bonded to the samples at the back using indium, which provides an Ohmic contact. The edges and backside of samples were sealed using epoxy to protect from contact to the electrolyte. Photocurrent measurements were carried out in a $400 \mathrm{~mL}$ aqueous solution of $0.25 \mathrm{M} \mathrm{Na}_{2} \mathrm{SO}_{4}$ buffered with phosphate buffered saline (PBS, Sigma) $(\mathrm{pH}=7.25)$ with three electrode configuration, including $\mathrm{ZnO} / \mathrm{Si}$ nanobroom array as a working photoelectrode (WE), Pt coil/mesh as a counter electrode (CE), and $\mathrm{Ag} / \mathrm{AgCl}(1 \mathrm{M} \mathrm{KCl})$ as a reference electrode (RE). The samples were illuminated with a constant light power intensity of $\sim 109 \mathrm{~mW} \mathrm{~cm}^{-2}$, from a solar simulator (Newport 67015) equipped with a $150 \mathrm{~W}$ Xenon lamp and a 1.5 AM filter. Note that the light power intensity was tuned to $100 \mathrm{~mW} \mathrm{~cm}^{-2}$ for the samples tested for the stability tests and catalyst effect (Figs. 7 and 8, as well as Figs. S3 and S6, see Supporting Information). The current versus potential/ time measurements were collected using a potentiostat (DY2300, Digi-Ivy). A scan rate of $5 \mathrm{mV} \mathrm{s}^{-1}$ was used for the current versus potential measurements. The time for each current density versus potential test was $10 \mathrm{~min}$ because the scan rate was $5 \mathrm{mV} \mathrm{s}^{-1}$ and the electrodes were scanned from -1.5 to $1.5 \mathrm{~V}$ versus $\mathrm{Ag} / \mathrm{AgCl} \mathrm{RE}$. The electrolyte was purged with $\mathrm{N}_{2}$ gas before start of each test for at least $5 \mathrm{~min}$ and a mild agitation was used during the PEC tests. Photocurrent stability tests were performed by collecting the 


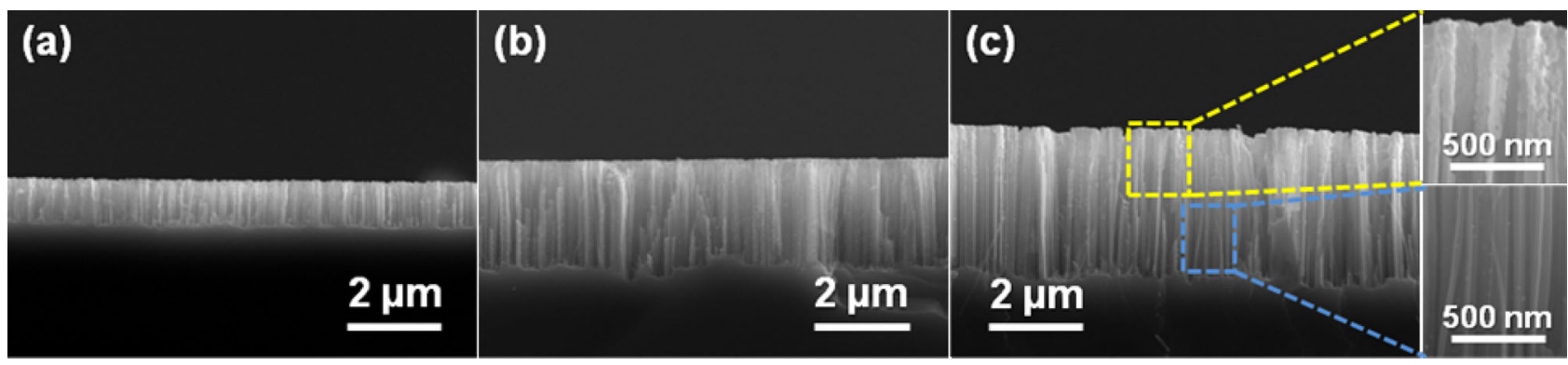

Figure 1 Cross-sectional SEM images of n-Si NW arrays etched for (a) $5 \mathrm{~min}$, (b) $10 \mathrm{~min}$, (c) $15 \mathrm{~min}$, and coated with ZnO seeding layer. The zoomed parts in (c) show the non-uniform coating of $\mathrm{ZnO}$ seeding layer on $\mathrm{Si}$ NWs.

electrode light current under constant light illumination and at a fixed biasing potential. The potentials versus normal hydrogen electrode (NHE) were calculated using $E_{\text {bias }}$ (vs. $\mathrm{NHE})=E_{\text {bias }}(\mathrm{vs} . \mathrm{Ag} / \mathrm{AgCl})+0.236 \mathrm{~V}$, where $0.236 \mathrm{~V}$ is the potential difference of $\mathrm{Ag} / \mathrm{AgCl} \mathrm{RE}(1 \mathrm{M} \mathrm{KCl})$ to the NHE at $25^{\circ} \mathrm{C}$ (Digi-Ivy, Inc., Austin, TX, USA).

3 Results and discussion Figure 1 shows crosssectional scanning electron microscopy (SEM) images of $n$ Si NW arrays with different wet etching times and coated with a $\mathrm{ZnO}$ seeding layer. The average $\mathrm{Si} \mathrm{NW}$ lengths are $\sim 900 \mathrm{~nm}, \sim 2.35 \mu \mathrm{m}$, and $\sim 3.21 \mu \mathrm{m}$ for $5 \mathrm{~min}, 10 \mathrm{~min}$, and 15 min etching times, respectively, resulting in an average etching rate of $\sim 200 \mathrm{~nm} \mathrm{~min}^{-1}$, which is consistent with that reported elsewhere [5]. The $\mathrm{ZnO}$ seeding layer does not uniformly cover the entire length of Si NWs, with larger thickness at the tips of Si NWs than other parts of NWs (see the zoomed SEM images in Fig. 1c for the top and bottom parts of Si NWs). In fact, at the bottom region of Si NWs, only $\mathrm{ZnO}$ nanoparticles or not a continuous film was observed (see the zoomed SEM image for the bottom part of NWs in Fig. 1c). The growth of ZnO NW stalls on the Si NW backbones for different growth times is illustrated in Fig. 2 . Longer $\mathrm{ZnO} \mathrm{NW}$ growth time results in bigger $\mathrm{ZnO} \mathrm{NWs}$ (larger diameter and longer length), which can clearly be seen by comparison between Fig. 2d, e, and f. Note that the $\mathrm{ZnO} \mathrm{NW}$ growth is only found at the tips of Si NWs and there is no growth on the sidewalls especially at the top parts of $\mathrm{Si} \mathrm{NWs}$, despite that the $\mathrm{ZnO}$ seeding layer covers uniformly the top portions of Si NWs (see Fig. 2d-f and g). The reason is not totally clear. It can be due to either a critical seeding layer thickness on the $\mathrm{Si}$ NW tips for $\mathrm{ZnO} \mathrm{NW}$ nucleation or a higher local $\mathrm{pH}$ due to initial growth reaction $[15,16]$. Low-magnification image in Fig. $2 \mathrm{~h}$ shows the large coverage of 3D $\mathrm{ZnO} / \mathrm{Si}$ nanobrooms. Note that different parts on samples were investigated and the formation of nanobrooms was throughout the entire area of samples. Figure 3a shows low-magnification bright field transmission electron microscopy (BF TEM) image of 3D $\mathrm{ZnO} / \mathrm{Si}$ nanobrooms on $\mathrm{Si}$ substrate with $10 \mathrm{~min} \mathrm{Si}$ NW backbones and $10 \mathrm{~min} \mathrm{ZnO} \mathrm{NW}$ stalls. Low-magnification dark field (DF) and BF scanning TEM (STEM) images of a single $\mathrm{ZnO} / \mathrm{Si}$ nanobroom are shown in Fig. $3 \mathrm{~b}$ and c. The average diameter of $\mathrm{Si} \mathrm{NW}$ backbone in this nanobroom is $\sim 100 \mathrm{~nm}$. Note that for the wet-etched Si NWs, the NWs are random in diameter (see Fig. 3a as an example) due to the nature of Ag-assisted electroless etching method. Highresolution high angle annular dark field (HAADF) STEM

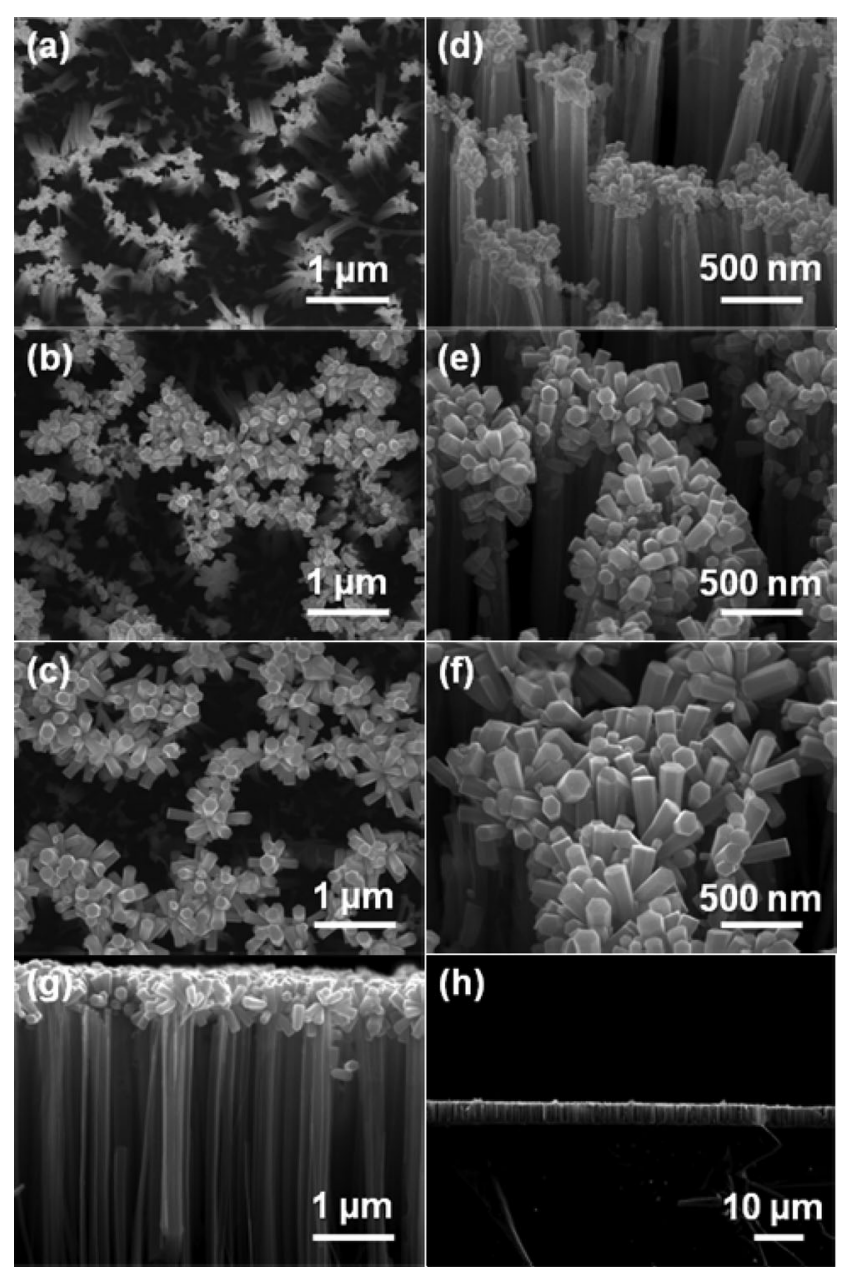

Figure 2 SEM images of $\mathrm{ZnO} / \mathrm{Si}$ nanobroom arrays with different ZnO NW growth times on 15 min Si NW backbone array. (a)-(c) and (d)-(f) show the top-view and tilted-view images of samples with 5, 10, and $15 \mathrm{~min} \mathrm{ZnO} \mathrm{NW}$ growth, respectively. (g) and (h) High- and low-magnification cross-sectional images of nanobrooms with $15 \mathrm{~min} \mathrm{ZnO}$ NW stalls grown on the Si NW backbone array with $15 \mathrm{~min}$ etching. 

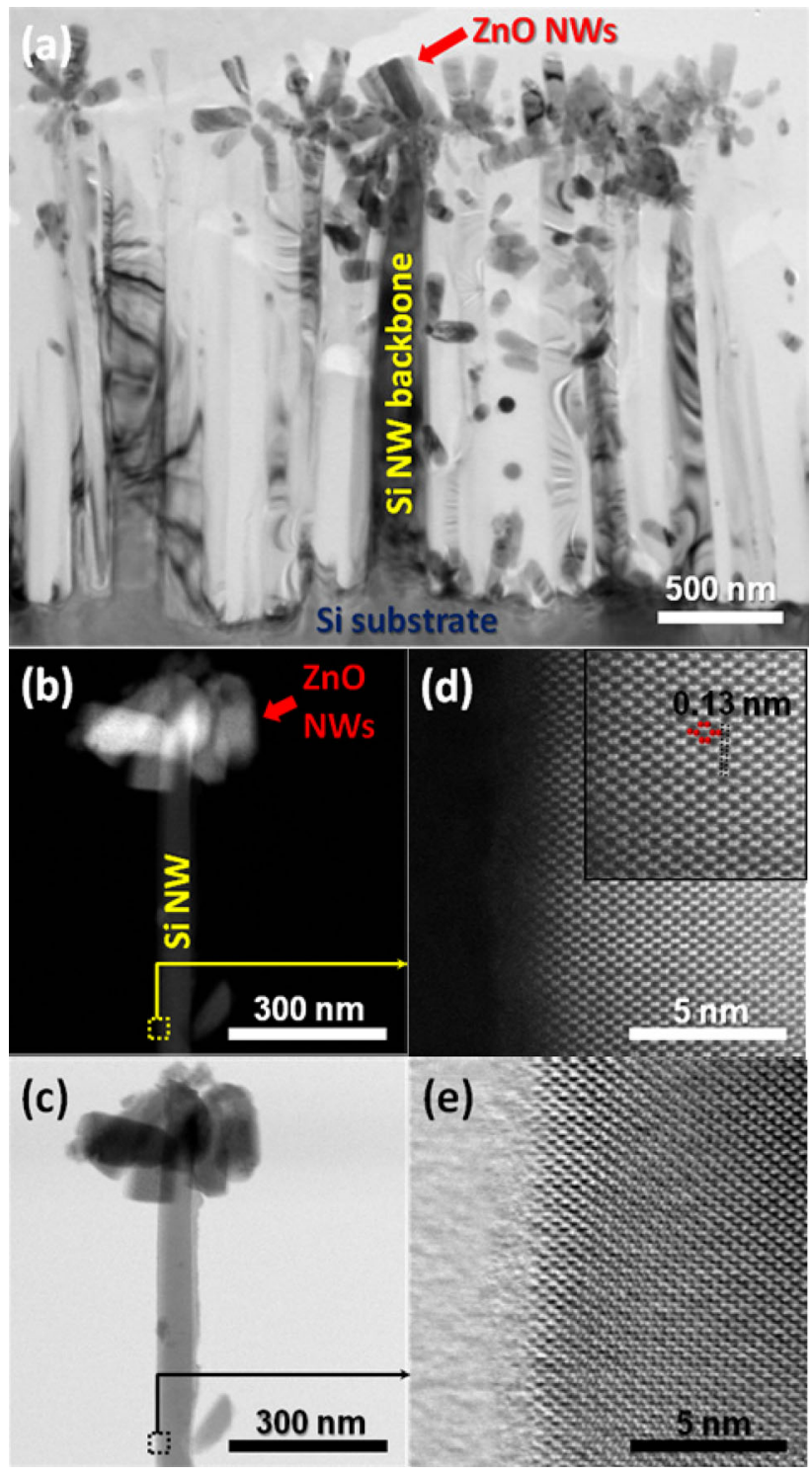

Figure 3 (a) Low-magnification $\mathrm{BF}$ TEM image of $\mathrm{ZnO} / \mathrm{Si}$ nanobroom arrays on Si substrate with $10 \mathrm{~min}$ Si NW backbones and $10 \mathrm{~min} \mathrm{ZnO} \mathrm{NW}$ stalls. Low-magnification DF STEM image (b) and BF STEM image (c) of a single $\mathrm{ZnO} / \mathrm{Si}$ nanobroom. (d, e) High-resolution annular STEM images of labeled parts in (b) and (c), respectively. (d) Inset shows the magnified STEM image with lattice spacing of corresponding $\mathrm{Si} \mathrm{NW}$, where red circle shows the diamond structure of Si.

image (Fig. 3d) for the highlighted position in Fig. 3b demonstrates defect-free and single-crystalline $\mathrm{Si}$ NW, which can also be seen in annular bright field (ABF) STEM image (Fig. 3e). The inset shows a lattice spacing of $0.13 \mathrm{~nm}$ down a 110 plane for the wet-etched Si NW. The red circle in the inset shows the diamond structure of $\mathrm{Si}$. The diffraction patterns for various positions along the Si NW backbone clearly show the single-crystalline nature along the entire length of the Si NW (see Fig. S2, Supporting Information).

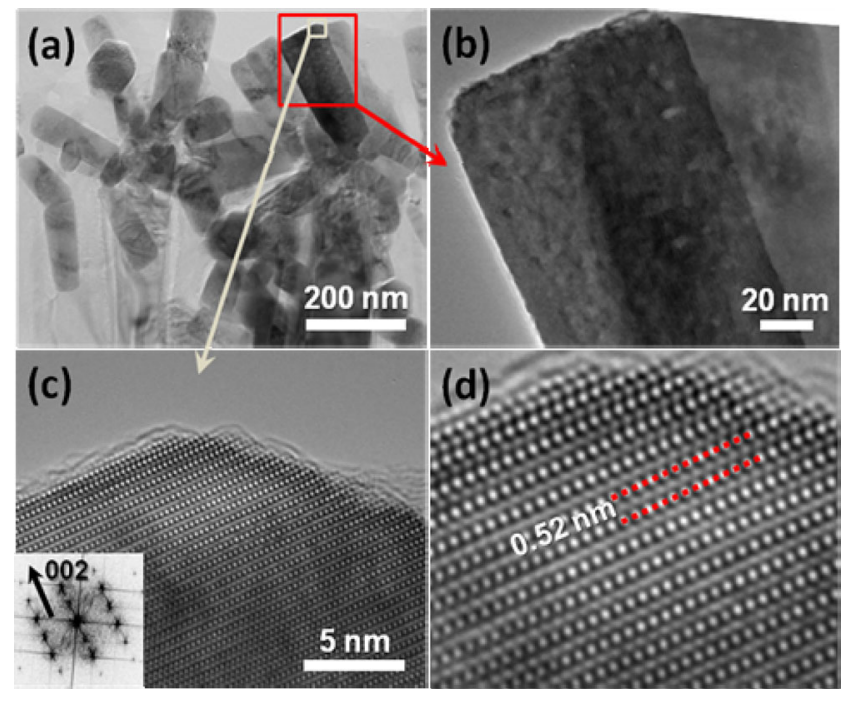

Figure 4 (a) Low-magnification BF TEM image of the top of the nanobrooms with $10 \mathrm{~min} \mathrm{Si}$ NW backbones and $10 \mathrm{~min} \mathrm{ZnO}$ NW stalls. (b) Zoom-in TEM image of a single ZnO NW. (c) Highresolution image of labeled part of a $\mathrm{ZnO} \mathrm{NW}$ tip and the corresponding FFT pattern indicating a $\mathrm{ZnO}$ NW growth direction of 002 orientation. (d) Corresponding noise-filtered TEM image of (c) with higher magnification showing the $\mathrm{ZnO}$ lattice spacing.

Low-magnification BF TEM image of ZnO NWs at the tip of Si NWs is shown in Fig. 4a. Figure 4b exhibits HRTEM image of a single $\mathrm{ZnO} \mathrm{NW}$ marked in Fig. 4a. The darker contrast shown in $\mathrm{ZnO} \mathrm{NW}$ is due to the overlap with another $\mathrm{ZnO}$ NW, also as seen in Fig. 4a. The HRTEM image in Fig. $4 \mathrm{c}$ demonstrates defect-free and single-crystalline wurtzite structure for the $\mathrm{ZnO} \mathrm{NW}$, which also shows very smooth surfaces. The calculated fast Fourier transform (FFT) pattern of this image, shown in the inset, illustrates the $\mathrm{ZnO} \mathrm{NW}$ growth direction of (002). The calculated lattice spacing is $0.52 \mathrm{~nm}$, shown in Fig. 4d, achieved from the modified image from noise-filtering of Fig. 4c.

Figure 5a shows the optical image of wet-etched n-Si NW arrays and arrays with $\mathrm{ZnO}$ NWs grown for various times. The as-etched Si NW samples are uniformly black in color over a large area indicating the uniform etching of the Si wafer. Sputtering a thin $\mathrm{ZnO}$ layer on the Si NW array changes its color to dark brown. There is a gradual color change toward more whiter/lighter with growth of larger $\mathrm{ZnO} \mathrm{NWs}$, in which sample with 15 min ZnO NWs looks gray (see "E" in Fig. 5a). The color change of nanobroom samples can be seen more clearly in Fig. 5b. Note that the optical images were taken in an angle less than $90^{\circ}$ compared to the surface of samples so that the difference in samples color can be seen (the view angle of Fig. 5a and b is slightly different). Obviously, this color change is due to the alteration of optical absorption, as shown in Fig. 5c. The Si NW array shows the highest absorption (though there is only a very small difference from that of the $\mathrm{ZnO}$-seeding-layer coated Si NW array) and the absorption decreases with 

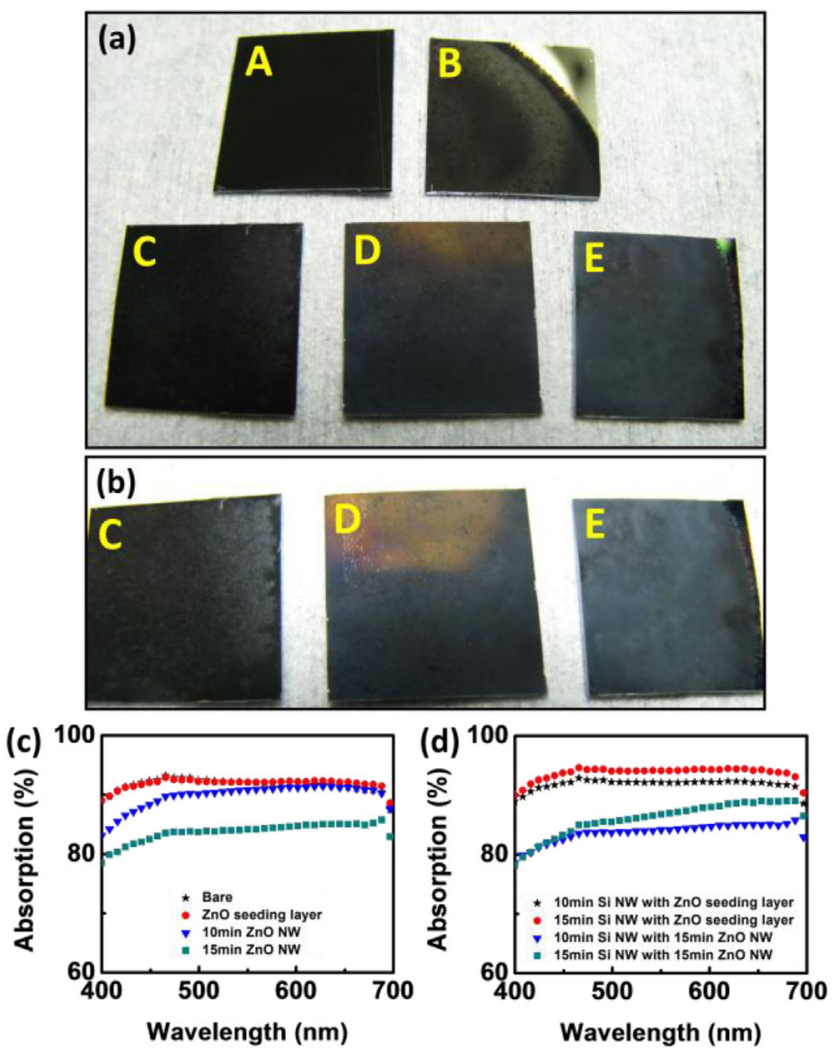

Figure 5 (a) Optical image of n-Si NW arrays (etched for 10 min) and with $\mathrm{ZnO}$ coating or $\mathrm{ZnO} \mathrm{NWs}$; A: bare Si NW array, B: with $\mathrm{ZnO}$ seeding layer coating, C: with $5 \mathrm{~min} \mathrm{ZnO} \mathrm{NW}$, D: with $10 \mathrm{~min}$ ZnO NW, E: with 15 min ZnO NW. (b) Optical image of samples C, $\mathrm{D}, \mathrm{E}$ with a slightly different view angle. Absorption spectra of (c) Si NW backbones (etched for $10 \mathrm{~min}$ ) and coated with $\mathrm{ZnO}$ seeding layer or NW stalls and (d) ZnO-seeding-layer coated n-Si NWs and nanobrooms with different Si NW etching times.

bigger $\mathrm{ZnO}$ NWs. The enhanced absorption of the vertical $\mathrm{Si}$ NW array is due to its excellent antireflection and light trapping effect properties [17-19]. Longer Si NWs lead to greater absorption due to improved light trapping [20], as shown in Fig. 5d. The reduced absorption for samples with $\mathrm{ZnO} \mathrm{NW}$ stalls can be due to increased scattering from $\mathrm{ZnO}$ NWs at the tip of Si NWs and the change in the refractive index gradient along the $\mathrm{Si}$ NWs due to $\mathrm{ZnO}$ coating.

The PEC performance of the nanobroom photoanodes in neutral solution $(\mathrm{pH}=7.25$ at room temperature) are shown in Fig. 6. The current density versus the externally applied potential for different Si NW etching times is presented in Fig. 6a. The photoanodic current increases with increasing Si NW length due to enhanced surface area for redox reaction and improved light absorption (see Fig. 5). The photoanodic current is comparable with that of $\mathrm{TiO}_{2}$ coated n-Si NW arrays considering the same length for $\mathrm{n}-\mathrm{Si}$ $\mathrm{NW}$ arrays [5]. The effect of $\mathrm{ZnO} \mathrm{NW}$ growth time on current is illustrated in Fig. 6b. Smaller ZnO NWs result in higher photoanodic current, which is probably due to better light absorption (see Fig. 5). Also, the smaller $\mathrm{ZnO}$ NWs in
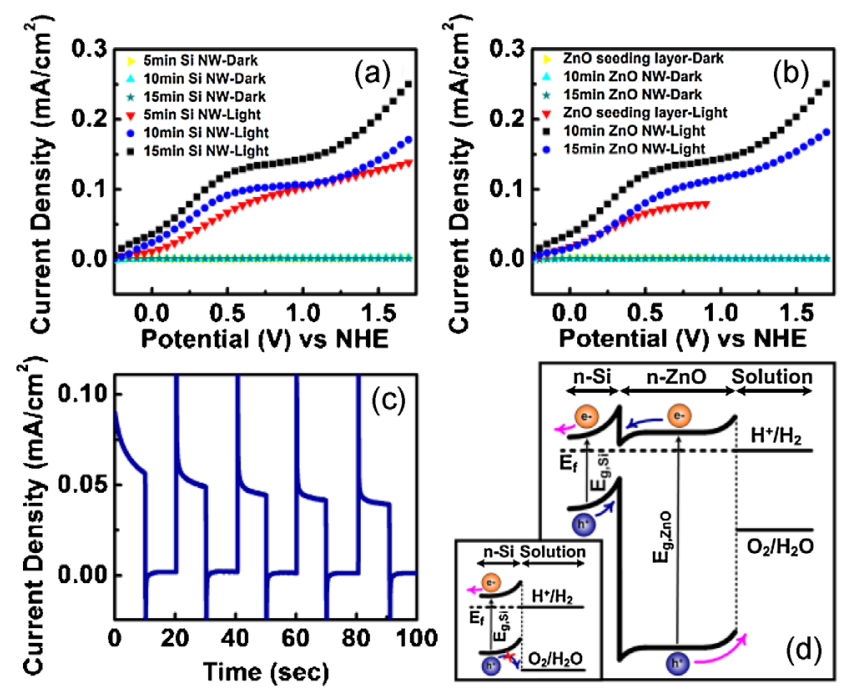

Figure 6 PEC performance and the working principle of the nanobroom photoelectrodes. Current density versus applied potential for the $\mathrm{ZnO} / \mathrm{Si}$ nanobroom photoelectrodes in neutral solution ( $\mathrm{pH}=7.25$ ); (a) 10 min grown $\mathrm{ZnO} \mathrm{NWs}$ on $\mathrm{n}$-Si NWs with different lengths (etching times) and (b) $\mathrm{ZnO} \mathrm{NW}$ stalls with different growth times on $15 \mathrm{~min}$ etched $\mathrm{n}-\mathrm{Si} \mathrm{NW}$ backbones. "Light" and "Dark" indicate the current density under illumination and at dark, respectively. Scan rates were $5 \mathrm{mV} \mathrm{s}^{-1}$ and the scan direction was from reversed to forward biases (from -0.25 to $1.72 \mathrm{~V}$ versus NHE). (c) Transient current density under chopped illumination for the nanobrooms with $15 \mathrm{~min}$ n-Si NW backbones and $15 \mathrm{~min} \mathrm{ZnO}$ NWs at biasing potential of $1.236 \mathrm{~V}$ versus NHE. (d) Proposed (approximate) energy band diagram of $n-\mathrm{ZnO} / \mathrm{n}-\mathrm{Si}$ NW heterojunction in contact with electrolyte under thermal equilibrium and illuminated with solar light having both UV and visible lights. The inset in (d) shows the approximate band diagram of n-Si NW in contact with solution at equilibrium and under the solar illumination.

nanobrooms may lead to more efficient charge separation and collection due to close proximity between photogenerated holes in $\mathrm{ZnO}$ and the $\mathrm{ZnO}$-electrolyte interface [21]. The n-Si NW array coated with $\mathrm{ZnO}$ seeding layer (i.e., zero $\mathrm{ZnO} \mathrm{NW}$ growth time) shows a lower photoanodic current compared to $\mathrm{ZnO} / \mathrm{Si}$ nanobroom heterostructures, agrees well with reported 3D branched NW photoelectrodes in which bare or ZnO-coated Si NW arrays show lower photocurrent than the branched NWs [8-10]. This can be due mainly to the increased surface area of $\mathrm{ZnO} / \mathrm{Si}$ nanobrooms than that of the $\mathrm{ZnO}$-seeding-layer coated Si NWs. Note that the dark currents are very small for all the tested samples. The transient current density under chopped illumination for the nanobrooms with $15 \mathrm{~min} \mathrm{Si}$ NW backbones and $15 \mathrm{~min} \mathrm{ZnO} \mathrm{NW}$ stalls at biasing potential of $1.236 \mathrm{~V}$ versus NHE is shown in Fig. 6c. As shown, there is a good switching behavior under switching on/off the light source. There are current overshoots (spikes) in the beginning of switching on/off, which can be attributed to accumulation of photo-generated carriers at the interface between NWs and the electrolyte, and the following 

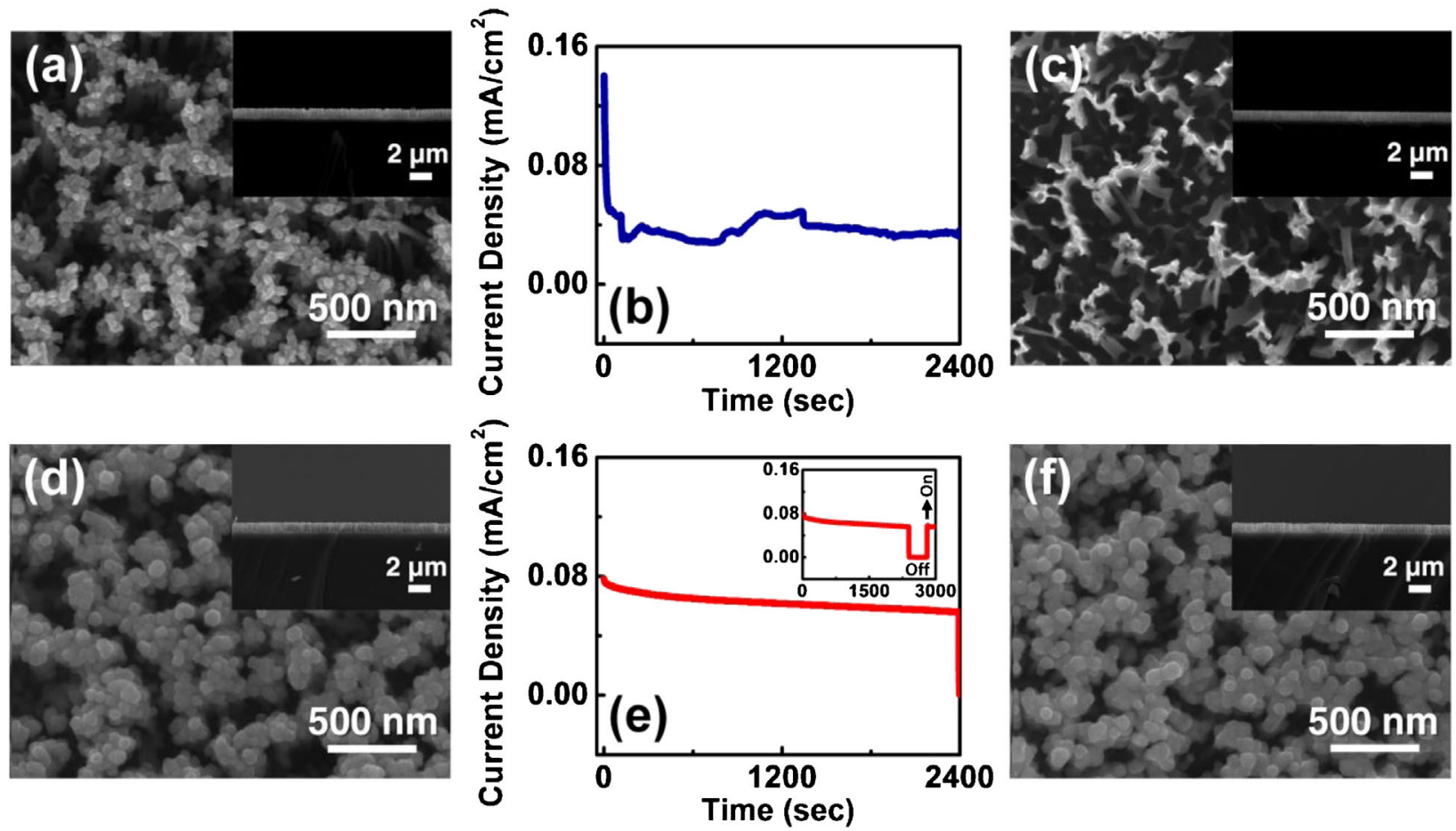

Figure 7 Stability performance of bare and $\mathrm{TiO}_{2}$-coated nanobrooms ( $5 \mathrm{~min} \mathrm{n}$-Si NW backbones with $5 \mathrm{~min} \mathrm{ZnO} \mathrm{NWs}$ ) in neutral solution ( $\mathrm{pH}=7.25$ ). (a)-(c) Bare nanobroom electrode; (a) SEM images of sample before any PEC and stability tests (as-grown sample), (b) anodic current density under constant light illumination versus time, (c) SEM images of sample after PEC tests and long-term stability test shown in (b). (d)-(f) $\mathrm{TiO}_{2}$-coated nanobroom sample; (d) SEM images of as-grown sample, (e) current density under fixed light illumination, (f) SEM images after PEC tests and long-term stability test in (e). The inset in (e) shows the stability test for longer time with one switching off/on the light illumination. The thickness of $\mathrm{TiO}_{2}$ was $10 \mathrm{~nm}$. The insets in $\mathrm{SEM}$ images show the low-magnification crosssectional images of corresponding samples in (a), (c), (d), and (f). The biasing potential was $1.236 \mathrm{~V}$ versus NHE for (b) and (e).

recombination until reaching a stationary current. Note also that the photoanodic current gradually decreases over time, which will be discussed in detail in the next discussion.

Figure $6 \mathrm{~d}$ shows the band diagram of the $\mathrm{ZnO} / \mathrm{Si}$ nanobroom heterostructure at the tips. The photo-generated electrons in the conduction band of $\mathrm{ZnO}$ recombine with the photo-generated holes in the valence band of $\mathrm{n}-\mathrm{Si}$ at the $\mathrm{n}-\mathrm{Si} /$ $\mathrm{n}-\mathrm{ZnO} \mathrm{NW}$ heterojunction, while the photo-generated holes in the $\mathrm{ZnO}$ travel to the $\mathrm{ZnO} \mathrm{NW}$ surface, oxidize water, and generate oxygen. Therefore, the band alignment and bending result in improved photoanodic behavior. Similar band alignment and explanation have been reported for the $\mathrm{n}-\mathrm{Si} / \mathrm{n}$ $\mathrm{TiO}_{2}$ core/shell NW heterostructure photoanodes [5]. Another key issue is the effect of the $\mathrm{ZnO}$ NW coverage on the Si NWs. Although the ZnO NWs do not cover the entire Si NW backbones, there is still an effective junction between $\mathrm{Si}$ and $\mathrm{ZnO}$ NWs at the tip of $\mathrm{Si}$ NWs (discussed above), and thus effective charge separation and enhanced photoactivity, similar to the reported flower-like $\mathrm{CuO} / \mathrm{ZnO}$ NW heterostructures where $\mathrm{CuO}$ NWs grown only at the tip of $\mathrm{ZnO} \mathrm{NWs}$ [22]. The photocurrent of $\mathrm{n}-\mathrm{ZnO} / \mathrm{n}-\mathrm{Si}$ nanobrooms is higher than the bare n-Si NW arrays due to the increased surface area and effective $\mathrm{Si}-\mathrm{ZnO}$ junction discussed above. In fact, the bare n-Si NW photoanode cannot provide much photocurrent due to the barrier for photo-generated holes to transfer to the electrolyte for water oxidation (inset of Fig. 6d). By applying a positive bias and increasing it, this barrier gradually reduces resulting in a small saturating photocurrent at high biases (see Fig. S3, Supporting Information). If the $\mathrm{ZnO} / \mathrm{Si}$ nanobroom array is exposed to only visible light (see Fig. S4, Supporting Information), the photo-generated carriers in Si NWs cannot lead to a significant current because the holes face a big barrier to transfer to the $\mathrm{ZnO}$ valence band and they eventually recombine with the photo-generated electrons. When the $\mathrm{ZnO} / \mathrm{Si}$ nanobrooms are illuminated with solar light having both UV and visible lights (see Fig. 6d), there are photo-generated carriers in both $\mathrm{Si}$ and $\mathrm{ZnO}$. Note that, in this case, Si can absorb both UV and visible lights. The effective $\mathrm{Si}-\mathrm{ZnO}$ junction (discussed above) assists that generated carriers in both $\mathrm{Si}$ and $\mathrm{ZnO}$ can more contribute to the current resulting in an improved photoanodic current than the bare $\mathrm{n}-\mathrm{Si}$ NWs. In fact, in this case, $\mathrm{ZnO}$ assists that photo-generated carriers in silicon can more contribute to the current (compared to the case with the bare n-Si NWs) revealing the significance of $\mathrm{ZnO} N W s$ in the improved PEC performance. Also comparing with the case with only visible light illumination, in this case $\mathrm{ZnO} \mathrm{NWs}$ assist that photogenerated carriers in silicon contribute to current while they 


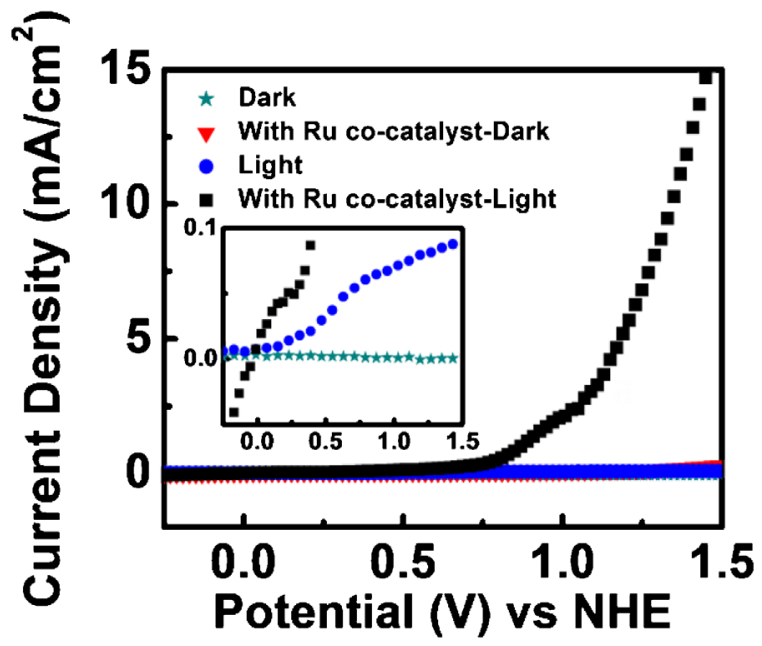

Figure 8 Current density versus applied potential of $\mathrm{TiO}_{2}$-coated nanobrooms (5 min $\mathrm{n}-\mathrm{Si} \mathrm{NW}$ backbones with $5 \mathrm{~min} \mathrm{ZnO} \mathrm{NWs}$ ) with and without $\mathrm{Ru}$ co-catalyst in neutral solution $(\mathrm{pH}=7.25)$. "Light" and "Dark" indicate the current density under illumination and at dark, respectively. The inset is the zoomed curves to show the level of current densities for the $\mathrm{TiO}_{2}$-coated nanobroom array in comparison to the light current of $\mathrm{TiO}_{2}$-coated nanobrooms with $\mathrm{Ru}$ co-catalyst. Scan rates were $5 \mathrm{mV} \mathrm{s}^{-1}$ and the scans direction was from reversed to forward biases (from -0.25 to $1.5 \mathrm{~V}$ versus NHE).

cannot contribute to a significant current under only visible light.

The $\mathrm{ZnO} / \mathrm{Si}$ nanobrooms are not stable over a long time and their photoanodic current decreases after a short time of a couple of minutes due to $\mathrm{Si}$ and $\mathrm{ZnO}$ instability in the electrolyte under light illumination and biasing condition. Therefore, the surface of nanobrooms is required to coat with a dissolution/corrosion-resistant layer in order to achieve long-term stability. $\mathrm{TiO}_{2}$ has been extensively applied as protection layer for the surface of different semiconductor photoelectrodes due to its high resistance to corrosion/ dissolution [23, 24]. Thus, to completely cover the surface of nanobrooms here, a thin $\mathrm{TiO}_{2}$ layer $(10 \mathrm{~nm})$ was deposited on them by ALD. The ALD is a gas phase deposition method and can deposit very conformal and ultrathin layers on complicated nanostructures such as nanobrooms here. As shown in Fig. S5c, see Supporting Information, the $10 \mathrm{~nm}$ $\mathrm{TiO}_{2}$ protection layer covers all surfaces of nanobrooms even the bottom of Si NW backbones. Figure $7 \mathrm{a}-\mathrm{c}$ show the stability performance of bare nanobrooms in neutral solution $(\mathrm{pH}=7.25)$. The nanobrooms in Fig. 7 are from 5 min n-Si NW backbones with 5 min ZnO NWs. As shown, the current under light illumination (light current) significantly decreases after a very short time (the light current of $0.14 \mathrm{~mA} \mathrm{~cm}^{-2}$ drops quickly), which is mainly due to the $\mathrm{ZnO}$ dissolution. The comparison between SEM images for before and after PEC and stability tests clearly demonstrates the $\mathrm{ZnO}$ decomposition as there is not any $\mathrm{ZnO} \mathrm{NW}$ and we can only see the Si NWs. The low-magnification cross- sectional SEM image in the inset of Fig. 7c shows that the Si NWs did not fall off and even their length is the same as that for before PEC and stability tests (see Fig. S5a and b in the Supporting Information). The surface of the Si NWs may also face some small changes such as very little oxidation or corrosion, which was hard to realize by the SEM imaging investigation. The stability test on the bare $\mathrm{n}-\mathrm{Si} \mathrm{NW}$ array also showed that the shape and morphology of n-Si NWs remained unchanged after the PEC and stability tests though there was photocurrent degradation overtime. Note that the current density versus potential measurements of nanobrooms cannot significantly affect by this lack of long-term stability because for the long-term stability test, the electrode faces intense fixed conditions (high biasing potential and current), while for the current density measurements, the biasing potential and current gradually change from low to high. Therefore, it is reasonable that the current density versus potential does not affect considerably. We have shown this for the $\mathrm{n}-\mathrm{ZnO} / \mathrm{p}-\mathrm{Si}$ branched NW photocathodes, in which different current scans provide similar currents while the photocurrent under fixed high bias reduce after a short time of a couple of minutes [9]. The stability performance of the $\mathrm{TiO}_{2}$-coated nanobrooms in neutral solution is shown in Fig. 7d-f. The light current of $\sim 0.08 \mathrm{~mA} \mathrm{~cm}^{-2}$ does not significantly change after long time. The current density at dark is constant over time as can be seen during the light off in the inset of Fig. 7e. Thus, there is a little reduction in photoanodic current (light current-dark current) over long time, which may be due to the charges trap in the $\mathrm{TiO}_{2}$ layer or at the interface [25]. After switching off/ on the light illumination during the stability test and after a long time (see the inset of Fig. 7e), the light current comes back to its original level before switching off, revealing the photoactivity of the nanobroom electrode after a long time stability test. Note that this was not the case for the bare nanobrooms as they lost their photoactivity after a short time. Interestingly, the shape and morphology of nanobrooms remain unchanged after the PEC and long stability tests revealing that the $\mathrm{TiO}_{2}$ coating layer well protects the surface of $\mathrm{Si}$ and $\mathrm{ZnO}$ NWs in which there is not any NW dissolution.

To enhance the oxygen evolution reaction (OER) at the surface of $\mathrm{TiO}_{2}$-coated $\mathrm{ZnO} / \mathrm{Si}$ nanobrooms, a metal catalyst can be used because there is OER kinetic limitation on the surface of bare semiconductors, especially $\mathrm{TiO}_{2}$ [2]. There are different metal OER catalysts such as $\mathrm{Ru}$, Ir, and Pt. One of the most efficient catalysts is $\mathrm{Ru}[24,26]$. The effect of $\mathrm{Ru}(\sim 5 \mathrm{~nm})$ OER catalyst on the PEC performance of $\mathrm{TiO}_{2}$-coated $\mathrm{ZnO} / \mathrm{Si}$ nanobrooms (5 min $\mathrm{n}-\mathrm{Si} \mathrm{NW}$ backbones with $5 \mathrm{~min} \mathrm{ZnO} \mathrm{NWs}$ ) in neutral solution is shown in Fig. 8. The Ru catalyst greatly increases the photoanodic current and also significantly reduces the anodic turn-on potential. The photoanodic current density (light current density-dark current density) of $\mathrm{Ru} / \mathrm{TiO}_{2}$-coated nanobroom array is $6.27 \mathrm{~mA} \mathrm{~cm}^{-2}$ at biasing potential of $1.236 \mathrm{~V}$ versus NHE, while it is only $0.08 \mathrm{~mA} \mathrm{~cm}^{-2}$ for the $\mathrm{TiO}_{2}$-coated nanobroom demonstrating the significance of using OER 
catalyst for efficient solar water splitting. The Ru catalyst also significantly improved the photocurrent of bare $\mathrm{n}-\mathrm{Si}$ NWs but with smaller photocurrent improvement than nanobrooms (see Fig. S6, Supporting Information). Note that the thickness of $\mathrm{TiO}_{2}$ for the $\mathrm{Ru} / \mathrm{TiO}_{2}$-coated nanobroom sample was a little less than $10 \mathrm{~nm}$; however, this thinner $\mathrm{TiO}_{2}$ layer did not significantly increase the sample photocurrent before Ru co-catalyst deposition in comparison with the photocurrent of the $\mathrm{TiO}_{2}$-coated nanobrooms with $10 \mathrm{~nm} \mathrm{TiO}_{2}$ (blue curve in the inset of Fig. 8) (in fact, the photocurrent increase was almost negligible). An efficient and cheap metal oxide OER catalyst such as $\mathrm{Ni}_{x} \mathrm{O}$ [27] can be used instead of metal OER catalyst in order to reduce the cost of photoelectrode.

4 Conclusions In summary, we reported the fabrication of $3 \mathrm{D} \mathrm{ZnO} / \mathrm{Si}$ nanobroom heterostructures using low-cost solution processing method and their application as PEC photoanodes for solar energy harvesting and solar fuel conversion. The morphology and atomic structure of the nanobrooms were characterized using high resolution SEM, TEM, and STEM. The nanobrooms consist of defect-free and single-crystalline $\mathrm{Si}$ NW backbones and $\mathrm{ZnO} \mathrm{NW}$ stalls with smooth surfaces. The PEC performance of the nanobroom photoelectrodes with different $\mathrm{Si}$ and $\mathrm{ZnO} \mathrm{NW}$ dimensions were studied and longer $\mathrm{Si} \mathrm{NW}$ backbones and smaller $\mathrm{ZnO} \mathrm{NW}$ stalls led to better light absorption and larger photoanodic current. The $\mathrm{ZnO} / \mathrm{Si}$ nanobroom electrodes showed much higher photoanodic current than the bare Si NW arrays. Long-term electrode stability was achieved using a thin $\mathrm{TiO}_{2}$ passivation layer protecting the NWs against decomposition. Large improvement in the photoanodic current and considerable reduction in the anodic turn-on potential were obtained by using $\mathrm{Ru}$ OER catalyst. These results show the promise of the use of 3D NW photoelectrodes from earth abundant materials by low-cost and simple fabrication methods for clean solar energy harvesting and conversion.

Acknowledgements D. W. acknowledges the financial support for this work by the Department of Energy (DOE DEFG36-08G018016) and the National Science Foundation (NSF ECCS0901113 and CBET1236155). D. W. thanks Dr. R. Rao and Dr. B. Fruhberger of Cal-IT2 of UCSD for their unconditional support. The structural analysis on $\mathrm{ZnO} / \mathrm{Si} \mathrm{NW}$ heterostructures using TEM and STEM were performed at the University of Michigan with the support of the Center for Solar and Thermal Energy Conversion, an Energy Frontier Research Center funded by the U.S. Department of Energy, Office of Science, Office of Basic Energy Sciences under Award Number DE-SC0000957. A. K. acknowledges S. Park and R. Anderson of UCSD NANO3 facilities for their timely and professional support. A. K. also acknowledges K. Madsen for his help for the PEC setup.

\section{References}

[1] M. Gratzel, Nature 414, 338 (2001).

[2] M. G. Walter, E. L. Warren, J. R. McKone, S. W. Boettcher, Q. Mi, E. A. Santori, and N. S. Lewis, Chem. Rev. 110, 6446 (2010).

[3] N. S. Lewis and D. G. Nocera, Proc. Natl. Acad. Sci. USA 103, 15729 (2006).

[4] H. B. Gray, Nature Chem. 1, 7 (2009).

[5] Y. J. Hwang, A. Boukai, and P. Yang, Nano Lett. 9, 410 (2009).

[6] Y. Tak, S. J. Hong, J. S. Lee, and K. Yong, J. Mater. Chem. 19, 5945 (2009).

[7] J. Su, L. Guo, N. Bao, and C. A. Grimes, Nano Lett. 11, 1928 (2011).

[8] K. Sun, Y. Jing, C. Li, X. Zhang, R. Aguinaldo, A. Kargar, K. Madsen, K. Banu, Y. Zhou, Y. Bando, Z. Liu, and D. Wang, Nanoscale 4, 1515 (2012).

[9] A. Kargar, K. Sun, Y. Jing, C. Choi, H. Jeong, G. Y. Jung, S. Jin, and D. Wang, submitted (2013).

[10] A. Kargar, K. Sun, Y. Jing, C. Choi, H. Jeong, Y. Zhou, K. Madsen, P. Naughton, S. Jin, G. Y. Jung, and D. Wang, Nano Lett. 13, 3017 (2013).

[11] K. Q. Peng, Y. J. Yan, S. P. Gao, and J. Zhu, Adv. Mater. 14, 1164 (2002).

[12] K. Sun, Y. Jing, N. Park, C. Li, Y. Bando, and D. Wang, J. Am. Chem. Soc. 132, 15465 (2010).

[13] M. Law, L. E. Greene, J. C. Johnson, R. Saykally, and P. Yang, Nature Mater. 4, 455 (2005).

[14] L. E. Greene, M. Law, J. Goldberger, F. Kim, J. C. Johnson, Y. Zhang, R. J. Saykally, and P. Yang, Angew. Chem. 42, 3031 (2003).

[15] A. Zainelabdin, G. Amin, S. Zaman, O. Nur, J. Lu, L. Hultman, and M. Willander, J. Mater. Chem. 22, 11583 (2012).

[16] S. K. Jung, S. Jeon, and K. Yong, Nanotechnology 22, 015606 (2011).

[17] K. Sun, A. Kargar, N. Park, K. N. Madsen, P. W. Naughton, T. Bright, Y. Jing, and D. Wang, IEEE J. Sel. Top. Quantum Electron. 17, 1033 (2011).

[18] A. Zhang, S. You, C. Soci, Y. Liu, D. Wang, and Y.-H. Lo, Appl. Phys. Lett. 93, 121110 (2008).

[19] C. Soci, A. Zhang, X.-Y. Bao, H. Kim, Y. Lo, and D. Wang, J. Nanosci. Nanotechnol. 10, 1430 (2010).

[20] E. Garnett and P. Yang, Nano Lett. 10, 1082 (2010).

[21] I. S. Cho, Z. Chen, A. J. Forman, D. R. Kim, P. M. Rao, T. F. Jaramillo, and X. Zheng, Nano Lett. 11, 4978 (2011).

[22] S. Jung and K. Yong, Chem. Commun. 47, 2643 (2011).

[23] A. Paracchino, V. Laporte, K. Sivula, M. Grätzel, and E. Thimsen, Nature Mater. 10, 456 (2011).

[24] Y. W. Chen, J. D. Prange, S. Dühnen, Y. Park, M. Gunji, C. E. D. Chidsey, and P. C. McIntyre, Nature Mater. 10, 539 (2011).

[25] A. Paracchino, N. Mathews, T. Hisatomi, M. Stefik, S. D. Tilley, and M. Grätzel, Energy Environ. Sci. 5, 8673 (2012).

[26] T. Reier, M. Oezaslan, and P. Strasser, ACS Catal. 2, 1765 (2012).

[27] K. Sun, N. Park, Z. Sun, J. Zhou, J. Wang, X. Pang, S. Shen, S. Y. Noh, Y. Jing, S. Jin, P. K. L. Yu, and D. Wang, Energy Environ. Sci. 5, 7872 (2012). 